\title{
A General Purpose Adaptive Fault Detection and Diagnosis Scheme for Information Systems with Superheterodyne Receivers
}

\author{
Dengwei Song, ${ }^{1,2}$ Hongmei Liu $\left(\mathbb{D},{ }^{1}\right.$ Le Qi ${ }^{1},{ }^{1,2}$ and Bo Zhou ${ }^{1,2}$ \\ ${ }^{1}$ School of Reliability and Systems Engineering, Beihang University, Xueyuan Road No. 37, Haidian District, Beijing, China \\ ${ }^{2}$ Science \& Technology on Reliability \& Environmental Engineering Laboratory, Xueyuan Road No. 37, Haidian District, Beijing, China \\ Correspondence should be addressed to Hongmei Liu; liuhongmei@buaa.edu.cn
}

Received 3 February 2018; Accepted 14 March 2018; Published 24 April 2018

Academic Editor: Minvydas Ragulskis

Copyright (c) 2018 Dengwei Song et al. This is an open access article distributed under the Creative Commons Attribution License, which permits unrestricted use, distribution, and reproduction in any medium, provided the original work is properly cited.

A superheterodyne receiver is a type of device universally used in a variety of electronics and information systems. Fault detection and diagnosis for superheterodyne receivers are therefore of critical importance, especially in noise environments. A general purpose fault detection and diagnosis scheme based on observers and residual error analysis was proposed in this study. In the scheme, two generalized regression neural networks (GRNNs) are utilized for fault detection, with one as an observer and the other as an adaptive threshold generator; faults are detected by comparing the residual error and the threshold. Then, time and frequency domain features are extracted from the residual error for diagnosis. A probabilistic neural network (PNN) acts as a classifier to realize the fault diagnosis. Finally, to mimic electromagnetic environments with noise interference, simulation model under different fault conditions with noise interferences is established to test the effectiveness and robustness of the proposed fault detection and diagnosis scheme. Results of the simulation experiments proved that the presented method is effective and robust in simulated electromagnetic environments.

\section{Introduction}

With the advent of the era of big data, data transmission is playing an increasingly important role. As a significant form of data in the wireless transmission of electronic information system, analog signal is irreplaceable in many specific circumstances because of its high accuracy of rate and simplicity of signal processing.

In information system, the superheterodyne receiver is a typical kind of analog signal receiving apparatus widely used in radars and all kinds of signal receivers. The superheterodyne receiver has the capability of converting highfrequency signals. Given its high sensitivity, frequency stability, and simpler structure, the superheterodyne receiver has essentially replaced all previous receiver designs and become a standard configuration for virtually all modern radio receivers, taking the use of frequency measuring receivers in electronic intelligence reconnaissance as an example [1]. Particularly in the military field, the superheterodyne receiver plays rather a more important role.
Serious receiver performance degradation is probably caused by faults of components in receivers. Due to the significance of superheterodyne receivers, the performance of whole information system and the quality of communication will be critically affected once superheterodyne receivers failed. Hence effective fault detection and diagnosis methods for superheterodyne receivers are vital for the enhancement of performance and the mission success rate. Accurate fault detection techniques can help to improve the availability of different superheterodyne receivers and fault detection is essential for initiating maintenance action to prevent total failure of the system. Meanwhile, efficient diagnosis is instrumental to disassembly and replacement malfunction component, and then the efficiency of equipment maintenance is improved. Finally, through synthetically consideration information on detection and diagnosis, utilization of superheterodyne receivers and the support of spare parts are rationally planned to guarantee satisfactory performance of information system. 


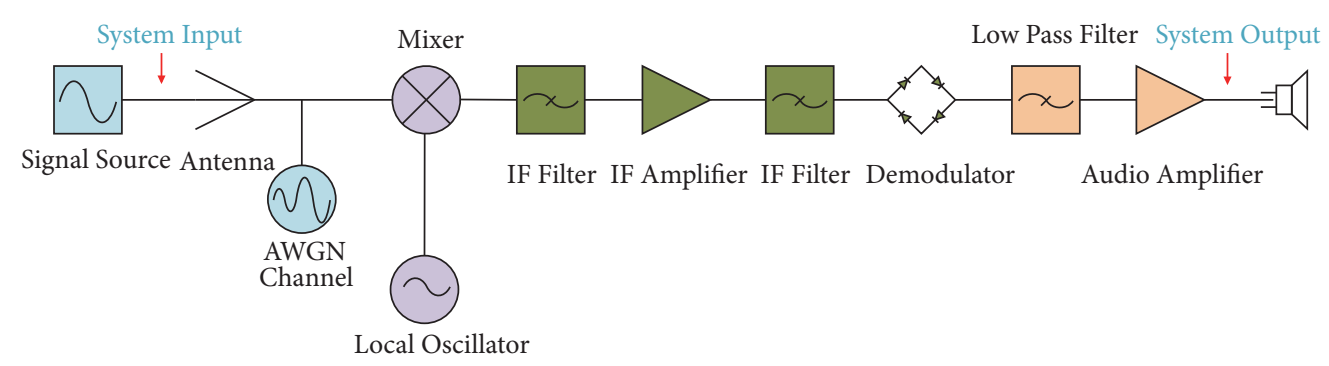

FIGURE 1: The block diagram of a superheterodyne receiver.

The implementation of fault detection and diagnosis in electromagnetic environment for superheterodyne receivers is a practical problem confronting electronic information equipment. However, there is little research known so far on fault detection and diagnosis for superheterodyne receivers under noise circumstance. Some relevant studies are as follows. In Binu and Kariyappa's survey, the approach of diagnosis of analog circuits was given and the machine learning-based approaches using artificial neural network as implementation were proposed [2]. Khanafseh and Pervan developed a general methodology to mitigate single receiver failures for architectures of carrier phase navigation [3]. Chen et al. proposed a fault diagnosis model for a radar receiver, built by applying multisignal flow graphs [4]. Mohsen and ElYazeed invented a diagnosis approach of analog circuits based on dictionary and the excellent effectiveness of proposed algorithm was demonstrated by soft fault simulation [5]. Nho et al. designed an algorithm and an operation boundary for fault detection of an onboard GNSS receiver [6].

However, most of these studies are just suitable for a single kind of receiver or some particular receiver types and therefore not applicable to receivers on a broader sense. To overcome this disadvantage, this study discusses a novel fault detection and diagnosis method which can be applied for most commonly used superheterodyne receiver types.

The method was inspired by fault detection schemes for control systems. In their work [7], Jayakumar and Das proposed a technique of fault detection for a flight control system based on Luenberger observer. Keliris et al. developed a nonlinear observer-based approach for distributed fault detection of a class of interconnected input-output nonlinear systems [8]. Pröll et al. presented a fault isolation method combining structural diagnostic ability analysis with observer-based residual generation [9]. These studies indicate that observerbased methods tend to have excellent performance in the fault detection problem for complex systems. Therefore, in this study, an observer-based fault detection and diagnosis method using adaptive thresholds were adopted in the information system with superheterodyne receivers to explore the application of the technique in a new field.

Observer is established to obtain the estimated output, and then the difference between the actual and estimated output is taken as the residual error, which carries a great deal of information of system. Fault detection is carried out by comparing the residual error with the adaptive threshold. A type of neural network is effective to meet the nonlinear requirements of observer and adaptive threshold generator.
Considering the nonlinear fitting capability of generalized regression neural network (GRNN), it is employed in this paper. Moreover, GRNN requires shorter training time to find optimal solution than BPNN [10].

From the point of view of pattern recognition, the process of fault diagnosis of a superheterodyne receiver is pattern classification for its operating status. Feature extraction of the residual error is the key point of fault diagnosis which determines whether the fault diagnosis is successful. To further process the residual error signal, time domain and frequency domain analysis are applied to extract fault features and then a classifier diagnoses the fault. A proper classifier also has a better effect on the result of fault diagnosis. Since the probabilistic neural network (PNN) is a widely used algorithm for classification applicable to nonlinear problems and high dimension applications with short training time [11], the diagnosis is realized using PNN.

To solve the aforementioned problems, a method that combines observer, adaptive threshold generator, and classifier based on PNN is proposed in order to realize the accurate fault detection and effective diagnosis, which is beneficial to improve the operation performance of the information system with superheterodyne receivers. Moreover, the method proposed in this study is featured with stronger engineering applicability and generality. It is more practical in communication fields compared with previous approaches since the only monitoring data required by the observer are the system input/output, while, on the other hand, the GRNN, employed as the fault observer, is able to describe virtually all kinds of nonlinear systems. In general, the method is robust to various noises under electromagnetic environments with noise interference, due to its adaptive nature.

The structure of this paper is organized as follows. The simulation model of a typical superheterodyne receiver is given in Section 2. In Section 3, the fault detection and diagnosis scheme is elaborated. In Section 4, the validity of the proposed method is proved using simulation data seeded with faults and the results of the experiments were given subsequently, and Section 5 concludes the paper with some perspectives.

\section{Modeling of a Typical Superheterodyne Receiver}

Figure 1 is the block diagram of a typical superheterodyne receiver consisting of local oscillator, mixer, bandpass filter, 


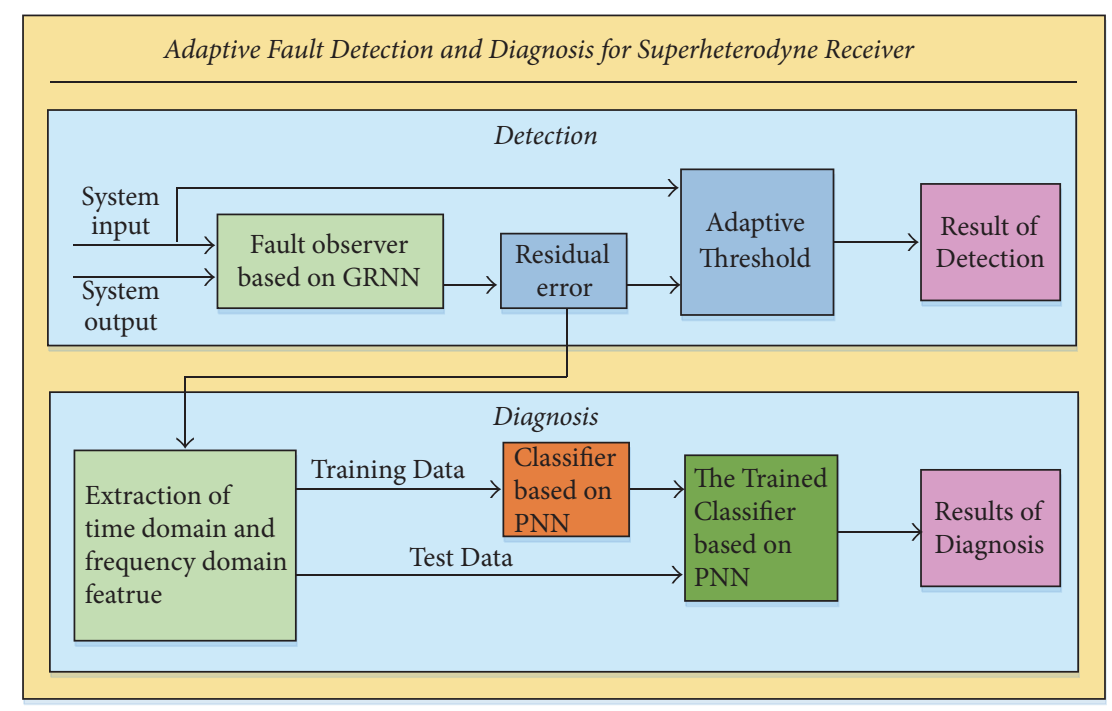

FIGURE 2: The adaptive fault detection and diagnosis for the superheterodyne receiver.

amplifier, demodulator, and additive white Gaussian noise (AWGN) channel.

In signal source system, the module is packaged into a subsystem, including original signal, carrier, and attenuator, to simulate the reception signal. The antenna collects the radio signal. Then the radio signal is processed as follows. First, the received signal is fed into a mixer where it is mixed with a sine wave known as the local oscillation signal. A local oscillator provides the mixing frequency that is variable for tuning the receiver to different stations. The frequency mixer does the actual heterodyning, which changes the incoming radio frequency signal. Second, the signal obtained by mixing is processed by intermediate frequency (IF) band-pass filter and amplifier successively. The IF band-pass filter provides the narrowband filter and amplifier provides most of the gain for the radio signal. Then the other IF filter processes the signal again to further eliminate the band noise. Third, the envelope detector demodulates signals from the filter and provides an output which is the envelope of the original signal. Last, the extracted signal is sent into a low-pass filter. Signal from filter is the system output after being amplified by audio amplifier.

Based on the composition and the principle of the superheterodyne receiver, the simulation model is established in Matlab/Simulink simulation environment. The details of the simulation model are as follows:

(a) In the signal source, the carrier frequency is $1000 \mathrm{kHz}$. The parameter of attenuator module is 0.1 . The module simulates attenuation caused by the transmission distance from the transmitter to the receiver.

(b) In AWGN channel, the means of random white noise is 0 , and the variance of random white noise is 0.001 . The noise is used to simulate the interference of electromagnetic environment.

(c) The local oscillation signal is from the voltagecontrolled oscillator controlled by the input voltage.
TABLE 1: Parameter of IF filter.

\begin{tabular}{lcc}
\hline Parameter & Unit & Value \\
\hline Filter order & Null & 1 \\
Center frequency & $\mathrm{Hz}$ & $465 \mathrm{k}$ \\
Bandwidth & $\mathrm{Hz}$ & $12 \mathrm{k}$ \\
\hline
\end{tabular}

Set up a slider gain module, and then the frequency of the local oscillator is controllable. The quiescent frequency of voltage-controlled oscillator is $465 \mathrm{kHz}$. The input sensitivity of voltage-controlled oscillator is $1000 \mathrm{~Hz} / \mathrm{V}$.

(d) Parameters of IF filters are shown in Table 1. The parameter of the IF amplifier is 20 . The upper and lower limits of envelope detector are set to inf and 0 . The parameter of the audio amplifier is 2 . The bandwidth of the low pass filter is $6 \mathrm{kHz}$.

\section{Fault Detection and Diagnosis for Superheterodyne Receivers}

Figure 2 shows the schematic diagram of the adaptive fault detection and diagnosis.

As is shown in the figure, in the phase of fault detection, an observer based on GRNN is fed with the system input and the system output. After training, the observer outputs an estimated value as a reference. Then residual error is obtained by comparing it with the actual output. Meanwhile, the other GRNN generates threshold that changes accordingly. The detection is finally realized by comparing the residual error and the adaptive threshold.

After fault detection, fault diagnosis is conducted based on the residual error generated by the fault observer. Three time domain parameters and three frequency domain parameters are extracted from the residual error signal. Finally, PNN is employed as the classifier to realize fault diagnosis. 


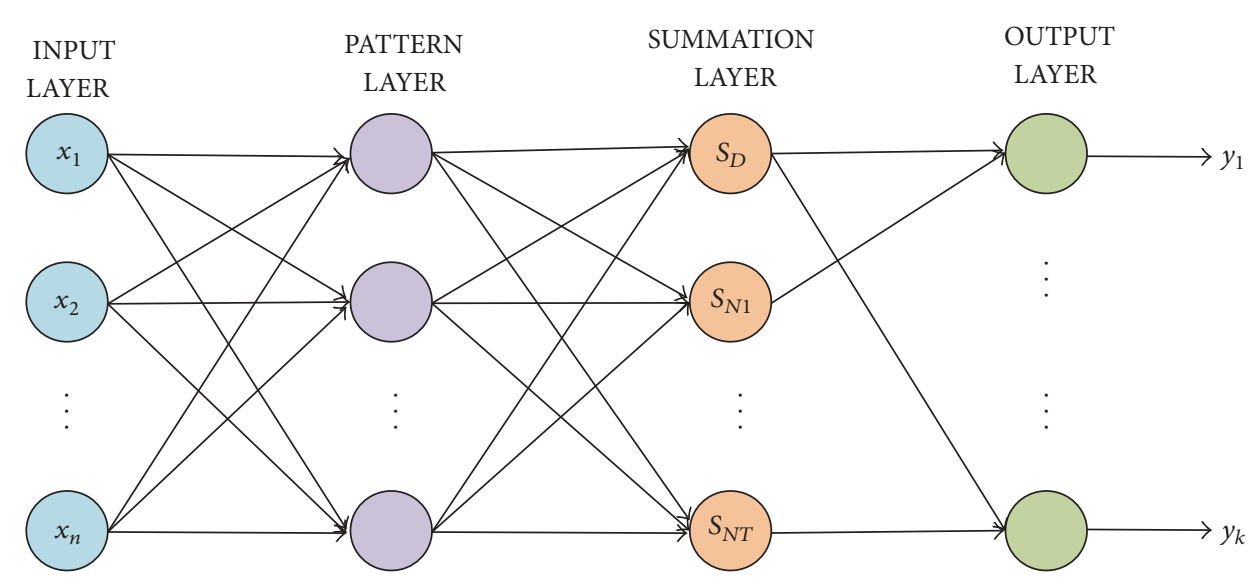

Figure 3: General configuration of GRNN.

\subsection{GRNN Observer Based Adaptive Fault Detection}

3.1.1. Generalized Regression Neural Network and Its Structure. Note that the fault detection is conducted in real-time electromagnetic environment where noise and interference will impact superheterodyne receivers' performance. GRNN is developed as an alternative to traditional neural network. As a feed-forward neural network based on nonlinear regression theory, GRNN has a simple and straightforward training algorithm which is significantly different from BPNN [12]. As shown in Figure 3, GRNN neural network includes four layers: an input layer, a pattern layer, a summation layer, and an output layer [13].

Assume that the network input is $X=\left[x_{1}, x_{2}, \ldots, x_{n}\right]^{T}$, and the corresponding network output is $Y=\left[y_{1}, y_{2}\right.$, $\left.\ldots, y_{k}\right]^{T}$. Each layer is presented in detail as follows.

(1) Input Layer. The number of neurons in the input layer is equal to the dimension of input feature vector. Each neuron is a simple distribution unit. The input of networks is transferred to the pattern layer directly.

(2) Pattern Layer. The number of neurons in the pattern layer is the total number of training samples $n$. Each neuron is related to different sample. The neural transfer function in the pattern layer is

$$
P_{i}=\exp \left[-\frac{\left(X-X_{i}\right)^{T}\left(X-X_{i}\right)}{2 \sigma^{2}}\right] \quad i=1,2, \ldots, n .
$$

In (1), $X$ is the system input. $X_{i}$ is the learning sample corresponding to the $i$ th neuron. Therefore, the output of neuron $i$ is the squared Euclidean distance between the input and the corresponding training sample.

(3) Summation Layer. There are two summation types in the summation layer.

The function of the first type is

$$
\sum_{i=1}^{n} \exp \left[-\frac{\left(X-X_{i}\right)^{T}\left(X-X_{i}\right)}{2 \sigma^{2}}\right] .
$$

This function sums out the output of all pattern layer neurons. The transfer function is

$$
S_{D}=\sum_{i=1}^{n} P_{i}
$$

The function of the second type is

$$
\sum_{i=1}^{n} Y_{i}\left[-\frac{\left(X-X_{i}\right)^{T}\left(X-X_{i}\right)}{2 \sigma^{2}}\right]
$$

Outputs of all pattern layer neurons were weighted and summed. $y_{i j}$ is the $j$ th element of the $i$ th output of training sample $Y_{i}$. The transfer function is

$$
S_{N j}=\sum_{i=1}^{n} y_{i j} P_{i} \quad j=1,2, \ldots, k .
$$

(4) Output Layer. The number of neurons in the output layer is equal to the dimension of output feature vector from training sample, which is $k$. The output of summation layer is divided in each neuron. The output of neuron $j$ is the $j$ th element of the network output:

$$
y_{j}=\frac{S_{N j}}{S_{D}} \quad j=1,2, \ldots, k .
$$

3.1.2. Design of the Fault Observer and Adaptive Threshold Generator. Design of a fault observer and an adaptive threshold generator using the method described in [14] is shown in Figure 4. Two GRNNs are utilized in this method. The first GRNN acted as an observer, which outputs the estimated system output. Then define the residual error by obtaining the deviation value between the estimated output and actual output. The other trained neural network is used as the adaptive threshold generator. The output datasets are composed of adaptive threshold values. Result of comparison between residual error and adaptive threshold can be applied to judge whether the system has fault or not.

In the training process, the system input and output are sent to the GRNN to obtain the observer. The residual error 


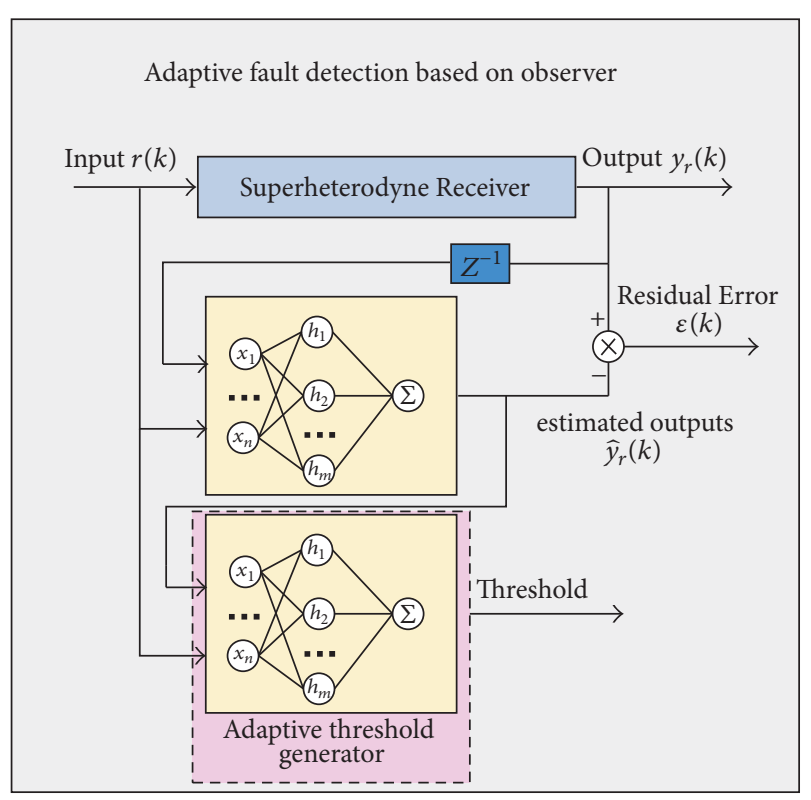

FIGURE 4: Fault detection based on GRNN observer.

is close to 0 when system works normally, while residual error increased when system is abnormal.

Aim to construct the relationship between the system input, output, and threshold. The training input datasets of the second neural network consist of the system input and output, and the training output data are the expected adaptive threshold calculated using (7).

$$
\text { threshold }=\varepsilon_{0}(k)+\beta .
$$

In (7), $\varepsilon_{0}(k)$ is the baseline residual error, which is acquired from observer under normal circumstances, and $\beta$ is the correction coefficient.

The observer and adaptive threshold generator can be employed to realize the fault detection after training. The residual error is below the threshold in normal status. While the system is in faulty status, the residual error exceeds the threshold.

3.2. Residual Error Analysis Based Fault Diagnosis. Residual error is the difference between the actual output and the estimated output; therefore the residual error carries large amount of fault information. Fault diagnosis is developed based on residual error.

\subsubsection{Feature Extraction in Time and Frequency Domain.} Feature extraction is a key issue in fault diagnosis. Properly extracted features can improve both diagnosis speed and accuracy.

The time domain analysis can characterize the energy and stability of signal over time. The frequency analysis can decompose the signal in detail. Considering the simplicity of time domain analysis and the adaptive ability of frequencydomain analysis, in this study, the feature of residual error is extracted by combining the time domain and frequency domain. Thereby the performance of system will be characterized as more comprehensive.

Three time domain parameters of signal, including peak value, root mean square, and average absolute value, are extracted. Suppose that a set of discrete data signal obtained by sampling is $x_{1}, x_{2}, \ldots, x_{n}$; equations of these parameters are as follows.

Peak value is

$$
\alpha=X_{\max }=\max \left\{\left|x_{i}\right|\right\} .
$$

Root mean square is

$$
\beta=X_{\mathrm{rms}}=\sqrt{\frac{1}{N} \sum_{i=1}^{N} x_{i}^{2}} .
$$

Average absolute value is

$$
\gamma=|\bar{X}|=\frac{1}{N} \sum_{i=1}^{N}\left|x_{i}\right| .
$$

Wavelet transformation (WT) and singular value decomposition (SVD) are general methods to extract the frequency domain feature. WT is an effective way to deal with signal. It can project a signal from time domain space to several frequency ranges, and the features of the dataset are implied in these frequency ranges. Signals are decomposed into a feature matrix and different frequency ranges can be obtained by using the WT. And the features usually appear in different frequency ranges [15]. SVD can be used to extract the prominent feature from all the frequency ranges. It can decompose a matrix into simple and meaningful pieces, which may contribute to the subsequent analysis. Matrix A decomposed by SVD can be expressed as follows:

$$
A=U S V^{T},
$$

where $U$ represents the left singular vector, $V$ denotes the right singular vector, and $S$ indicates diagonal matrix whose elements in the main diagonal are singular values.

The samples belonging to the same category will have the similar singular values and samples with different categories decomposed by SVD will obtain diverse singular values [16]. Hence, the singular values can be utilized to represent a signal.

After the analysis in time and frequency domain, the eigenvector of superheterodyne receivers is composed of time domain and frequency domain features.

3.2.2. PNN Based Fault Diagnosis. In this study, PNN is employed as classifier for fault diagnosis. As a radial basis function neural network, a PNN neural network is a powerful algorithm for classification with its theory based on Bayes minimum risk criteria (Bayesian decision theory) [17]. Due to its concise training and strong classification ability, the PNN has widely applications for fault diagnosis in practical applications [18]. Compared with BP neural network, the advantage of a PNN is that the topology, connection weights, 


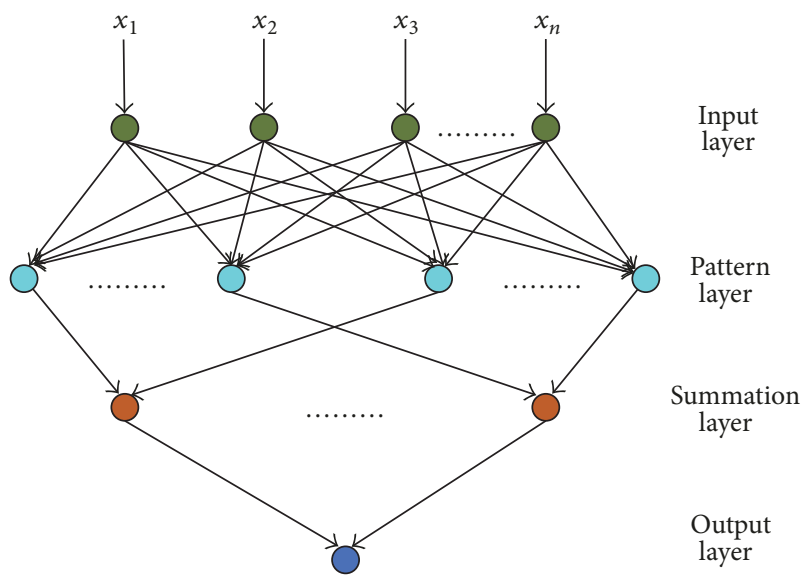

FIGURE 5: General configuration of PNN.

TABLE 2: Injected fault modes in simulation model.

\begin{tabular}{lcc}
\hline Number & Fault & Details \\
\hline 1 & Amplifier fault & Amplifier gain reduction fault \\
2 & Oscillator fault & Local oscillator bias fault \\
3 & Filter fault & Filter bandpass reduction fault \\
\hline
\end{tabular}

and thresholds can be set immediately when training samples are attainable [19].

The general structure of a PNN model is shown in Figure 5. The model contains 4 layers: input layer, the pattern layer, the summation layer, and the output layer [20].

The input layer introduced the eigenvector into network. Pattern layer calculates the distance between the unknown input and the training sample. Then distance transformed by activation function, which is Gaussian function, is the output of pattern layer. The number of neurons in summation layer is equal to the number of failure modes. Each neuron sums the probability of one kind of fault. According to the estimated probability, the output layer chooses a neuron in summation layer with the highest probability as the output of the neuro network.

In the process of diagnosis, the training data is used to train the PNN; the weights of neuro network and other parameters are created in the learning stage. In the recalling stage, the PNN is utilized as a classifier to identify the system status.

\section{Case Study}

4.1. Simulation Parameters and Fault Injection. A sine signal is used as the input signal is in the simulation. The amplitude is 1 and the system input frequency is $100 \mathrm{~Hz}$.

According to the statistics of historical maintenance data, main fault types of a superheterodyne receiver include amplifier fault, oscillator fault, and filter fault, which are fed into the simulation model to test the effectiveness of the proposed approach.

The fault modes listed in Table 2 were introduced into the simulation model by changing several specific parameters of

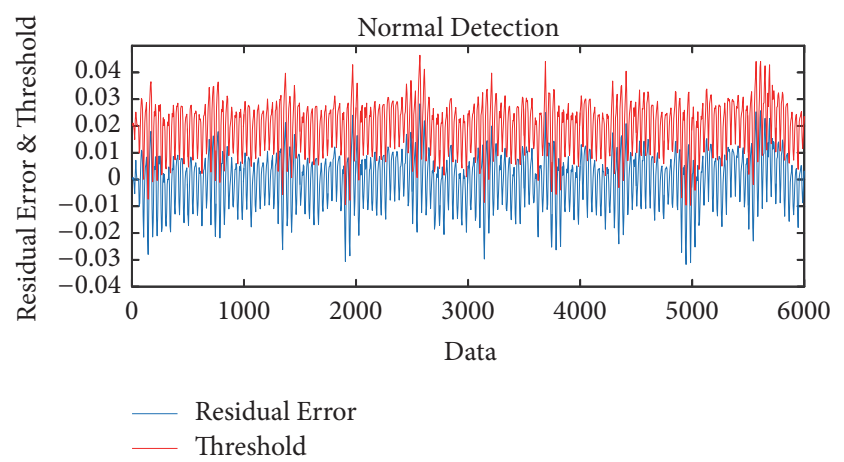

FIGURE 6: Detection result under normal status.

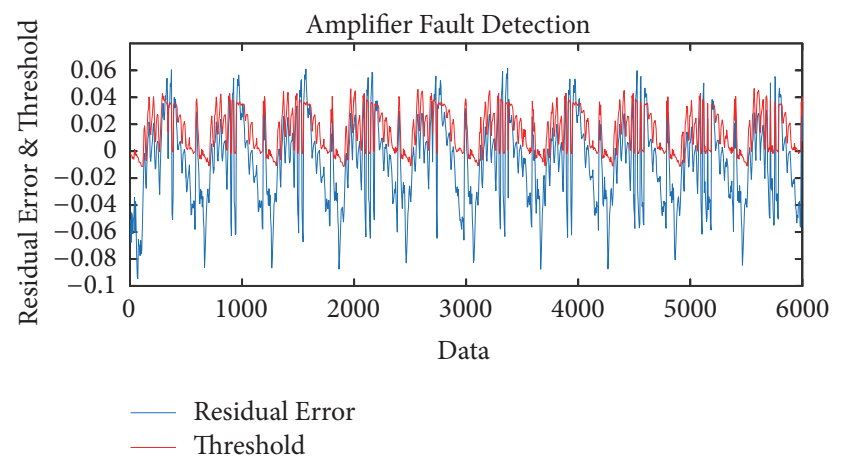

Figure 7: Detection result for amplifier fault.

the fault components. The details of fault injection are listed in Table 3.

The simulation duration is $0.01 \mathrm{~s}$, and the sampling rate is $600 \mathrm{~K} / \mathrm{s}$. Data of normal status and three kinds of fault status were collected. In each case there are 6000 data points of system input and 6000 data points of system output.

4.2. Fault Detection and Results Analysis. For fault detection, normal data of system input and output are used to train the observer based on the first GRNN. The system input and the system output of the previous step are taken as the input sample of training data. The output sample of training data is the actual system output. The function spread of the first GRNN neural network is set to 0.01 .

Normal data are used to train the second GRNN to generate the adaptive threshold as well. The system input and the estimated system output are taken as the input sample of training data. The output sample of training data is the expected adaptive threshold which is the summation of the baseline residual and $\beta$. Through multiple tests combined experience, the value of $\beta$ is set to 0.1. The function spread of the second GRNN neural network is set to 0.01 .

The structure and parameters of the GRNN neural networks are determined after training.

Four tests were carried out to validate the effectiveness of the fault detection scheme. The results are shown in Figures 6-9. In each figure, the blue curve shows the residual error, and the red curve is the adaptive threshold. 
TABLE 3: Fault injection details.

\begin{tabular}{lccccc}
\hline Test number & Fault mode & Fault component & Changed parameter for fault injection (unit) & Parameter (normal) & Parameter (fault) \\
\hline 1 & Normal & - & - & - \\
2 & Amplifier fault & Electronic amplifier & Gain & 20 & 17 \\
3 & Oscillator fault & Local oscillator & Quiescent frequency (kHz) & 465 & 480 \\
4 & Filter fault & Intermediate frequency filter & Bandwidth (kHz) & 12 & 1 \\
\hline
\end{tabular}

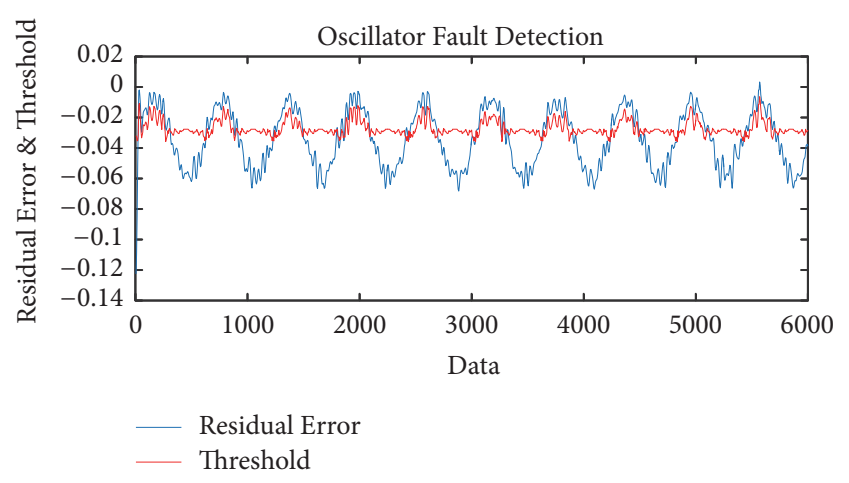

FIGURE 8: Detection result for oscillator fault.

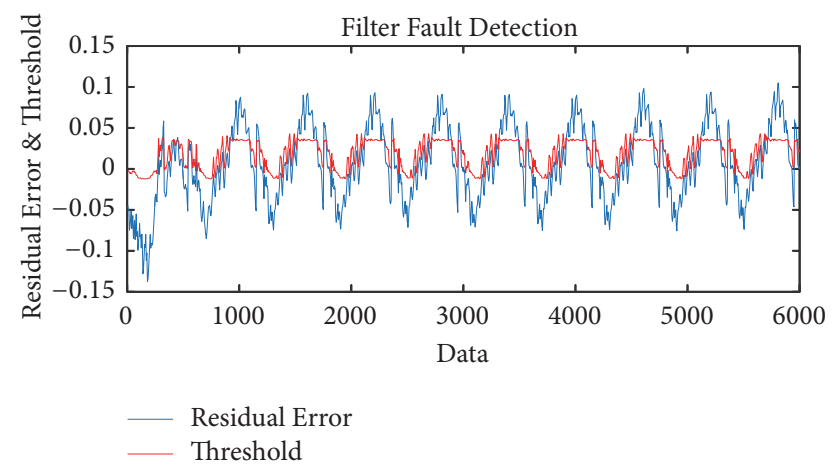

FIGURE 9: Detection result for filter fault.

In test 1 , the superheterodyne receiver works normally. The result of detection is shown in Figure 6. The residual error was close to zero and below the threshold; therefore the results indicate that the system is under normal circumstances.

In test 2, an electronic amplifier fault was injected to the system. The result of detection is shown in Figure 7. The residual error became greater and exceeded the threshold; therefore, the results indicate that fault occurred in the system.

In test 3 , an oscillator fault was injected to the system. The result of detection is shown in Figure 8. The residual error is beyond the threshold; therefore, the results indicate that fault occurred in the system.

In test 4 , a filter fault was injected to the system. The result of detection is shown in Figure 9. The residual error increased significantly and exceeded the threshold; therefore, the results indicate that fault occurred in the system.
TABLE 4: Training eigenvectors for fault diagnosis.

\begin{tabular}{lcc}
\hline Pattern & Sample size & Label data \\
\hline Normal & 210 eigenvectors & 1 \\
Filter fault & 210 eigenvectors & 2 \\
Amplifier fault & 210 eigenvectors & 3 \\
Oscillator fault & 210 eigenvectors & 4 \\
\hline
\end{tabular}

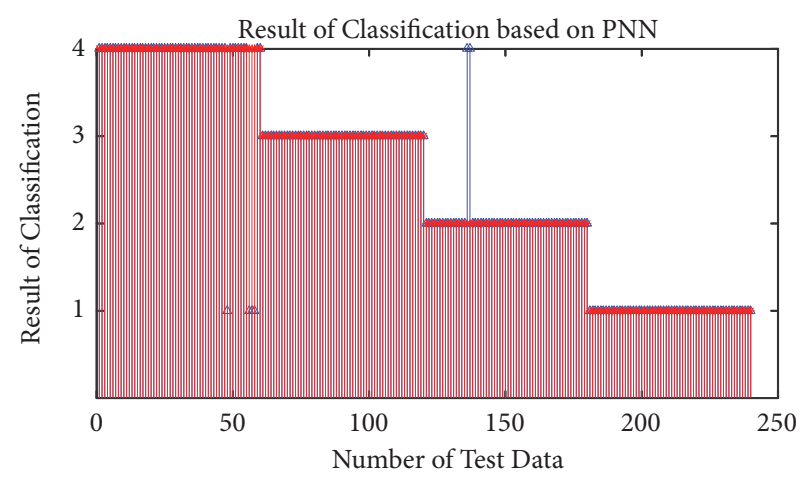

Figure 10: Result of Classification based on PNN.

As indicated by the simulation results, the residual error exceeded the threshold when the system worked abnormally under the interference of environment noise factors.

4.3. Fault Diagnosis and Results Analysis. For fault diagnosis, the time domain features, including the average absolute value, the root mean square, the peak value, and three frequency domain features processed by WT-SVD, are obtained from residual error. Data were preprocessed and one eigenvector including 6 features was extracted from each of 600 samples of residual error signals. 270 eigenvectors were extracted from each residual error signal and totally 1080 eigenvectors were obtained.

To train the PNN, 840 eigenvectors of each fault mode were selected to form the training data, and their labels were set as shown in Table 4 . The function spread of PNN was set to 0.075 .

After the training of PNN, the other 240 eigenvectors were employed as the test data to verify the effectiveness of the proposed method. Figure 10 shows the results of fault diagnosis. In Figure 10, the red points are the actual labels of the test data, while the blue points are the predicted labels by PNN.

Number 1 to number 60 are the normal data. Number 61 to number 120 are the filter fault data. Number 121 to number 180 are the amplifier fault data. Number 181 to number 240 are 
the oscillator fault data. In the test of normal data, the PNN classification result of 4 test samples did not match the actual label. In the test of amplifier fault data, the PNN classification result of all test samples matched the actual label. In the test of oscillator fault data, the PNN classification result of 2 test samples did not match the actual label. In the test of filter fault data, the PNN classification result of all test samples matched the actual label.

In total, there are 6 test samples of the misjudgment of the fault diagnosis out of 240 test samples, and the corresponding diagnosis accuracy was $97.5 \%$.

\section{Conclusion}

In this paper, a general purpose fault detection and diagnosis method was proposed for information systems with superheterodyne receivers. By establishing the observer and adaptive threshold generator based on GRNNs, the faults of superheterodyne receiver are detected by the variation of residual error. The residual error is then used to extract fault features and PNN is employed as the fault classifier to diagnose the fault type. The proposed method was verified by simulation experiments considering noise interferences. The result indicates that the proposed method can effectively detect superheterodyne receiver faults and diagnose fault modes accurately. Meanwhile, the adaptive threshold has strong adaptive ability for noise interferences.

The advantage of the proposed approach lies in that it is applicable not only to the superheterodyne receiver but also to more complex signal receiving systems of similar designs in which the transfer functions are difficult to obtain. It also outweighs other previous methods due to its strong robustness to environmental noises and interferences and enables a higher mission success rate.

However, future work needs to be done to make the proposed method more practical. First, the data from a lab testing of actual superheterodyne receiving system will be applied instead of simulation model to further verify the effectiveness of the proposed method. Second, the classifier needs to be developed to realize the diagnosis in the situation with multiple failures.

\section{Conflicts of Interest}

The authors declare that they have no conflicts of interest.

\section{Acknowledgments}

This research was supported by the National Natural Science Foundation of China [Grants nos. 51605014, 51105019, and 51575021], the Technology Foundation Program of National Defense [Grant no. Z132013B002], and the Fundamental Research Funds for the Central Universities [Grant no. YWF16-BJ-J-18].

\section{References}

[1] Y. Qiang, B. Daping, H. Bing, and X. Lianghao, "Jamming technology study of superheterodyne receiver," Fire Control \& Command Control, vol. 10, pp. 56-59, 2015.
[2] D. Binu and B. S. Kariyappa, "A survey on fault diagnosis of ana$\log$ circuits: Taxonomy and state of the art," AE ̈U - International Journal of Electronics and Communications, vol. 73, pp. 68-83, 2017.

[3] S. Khanafseh and B. Pervan, "Detection and mitigation of reference receiver faults in differential carrier phase navigation systems," IEEE Transactions on Aerospace and Electronic Systems, vol. 47, no. 4, pp. 2391-2404, 2011.

[4] S.-J. Chen, K. Lian, and H.-J. Wang, "Fault diagnosis method of radar receiver using multi-signal flow graphs model," Dianzi Keji Daxue Xuebao/Journal of the University of Electronic Science and Technology of China, vol. 38, no. 1, pp. 87-91, 2009.

[5] A. A. K. Mohsen and M. F. A. El-Yazeed, "Selection of input stimulus for fault diagnosis of analog circuits using ARMA model," AEÜ - International Journal of Electronics and Communications, vol. 58, no. 3, pp. 212-217, 2004.

[6] H. Nho, J. Ahn, S. Sung, H. Jun, C. Yeom, and Y. Lee, "Research on Algorithm and Operation Boundary for Fault Detection of Onboard GNSS Receiver," Journal of the Korean Society for Aeronautical \& Space Sciences, vol. 40, no. 2, pp. 171-177, 2012.

[7] M. Jayakumar and B. B. Das, "Fault detection, isolation and reconfiguration in presence of incipient sensor faults in an electromechanical flight control actuation system," in Proceedings of the 2006 IEEE International Conference on Industrial Technology, ICIT, pp. 92-97, India, December 2006.

[8] C. Keliris, M. M. Polycarpou, and T. Parisini, "A robust nonlinear observer-based approach for distributed fault detection of input-output interconnected systems," Automatica, vol. 53, pp. 408-415, 2015.

[9] S. Pröll, F. Jarmolowitz, and J. Lunze, "A comprehensive observer-based fault isolation method with application to a hydraulic power train," IFAC-PapersOnLine, vol. 49, no. 11, pp. 547-554, 2016.

[10] D. F. Specht, "A general regression neural network," IEEE Transactions on Neural Networks and Learning Systems, vol. 2, no. 6, pp. 568-576, 1991.

[11] D. F. Specht, "Probabilistic Neural Networks and the Polynomial Adaline as Complementary Techniques for Classification," IEEE Transactions on Neural Networks and Learning Systems, vol. 1, no. 1, pp. 111-121, 1990.

[12] R. Rooki, "Application of general regression neural network (GRNN) for indirect measuring pressure loss of HerschelBulkley drilling fluids in oil drilling," Measurement, vol. 85, pp. 184-191, 2016.

[13] H. Bendu, B. B. V. L. Deepak, and S. Murugan, "Application of GRNN for the prediction of performance and exhaust emissions in HCCI engine using ethanol," Energy Conversion and Management, vol. 122, pp. 165-173, 2016.

[14] H. Yuan, C. Lu, J. Ma, and Z.-H. Chen, "Neural network-based fault detection method for aileron actuator," Applied Mathematical Modelling, vol. 39, no. 19, pp. 5803-5815, 2015.

[15] O. Daoud, Q. J. Hamarsheh, and S. Saraireh, "PAPR reduction based on entropy wavelet transform for Sniffer Mobile Robot," $A E \ddot{U}$ - International Journal of Electronics and Communications, vol. 68, no. 12, pp. 1165-1172, 2014.

[16] Q. Zhang, Y. Wang, M. D. Levine, X. Yuan, and L. Wang, "Multisensor video fusion based on higher order singular value decomposition," Information Fusion, vol. 24, pp. 54-71, 2015.

[17] D. F. Specht, "Probabilistic neural networks," Neural Networks, vol. 3, no. 1, pp. 109-118, 1990. 
[18] H. Malik and S. Mishra, "Application of Probabilistic Neural Network in Fault Diagnosis of Wind Turbine Using FAST, TurbSim and Simulink," in Proceedings of the 2nd International Symposium on Computer Vision and the Internet, VisionNet '15, pp. 186-193, India, August 2015.

[19] S. R. Mohanty, P. K. Ray, N. Kishor, and B. K. Panigrahi, "Classification of disturbances in hybrid DG system using modular PNN and SVM," International Journal of Electrical Power \& Energy Systems, vol. 44, no. 1, pp. 764-777, 2013.

[20] J. Jing, H. Liu, and C. Lu, "Fault diagnosis of electro-mechanical actuator based on WPD-STFT time-frequency entropy and PNN," in Proceedings of the 28th International Conference on Vibroengineering, pp. 130-135, China, October 2017. 


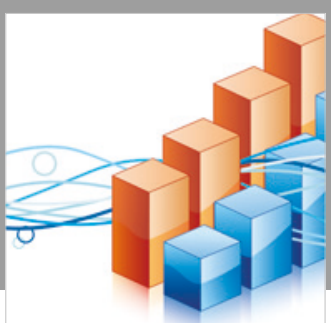

Advances in

Operations Research

\section{-n-m}
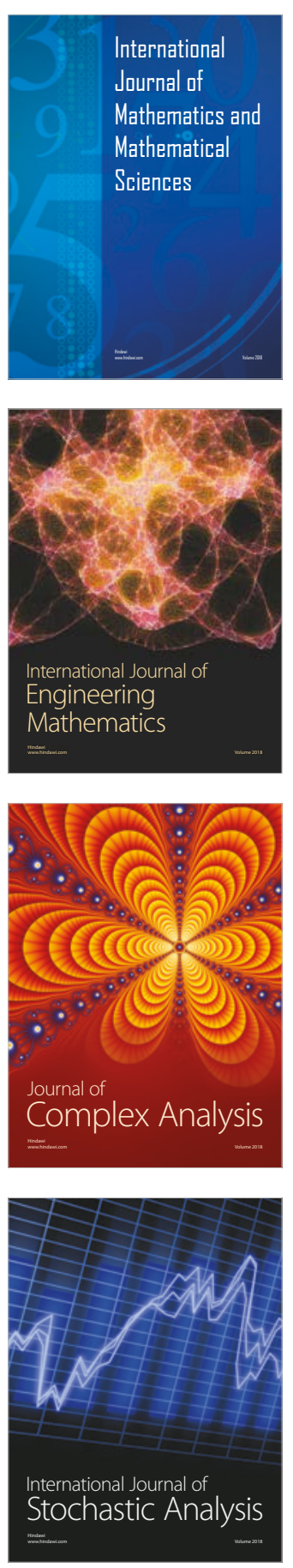
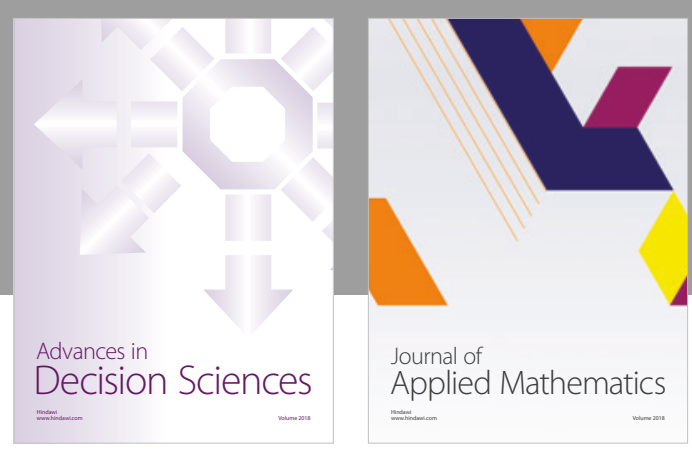

Journal of

Applied Mathematics
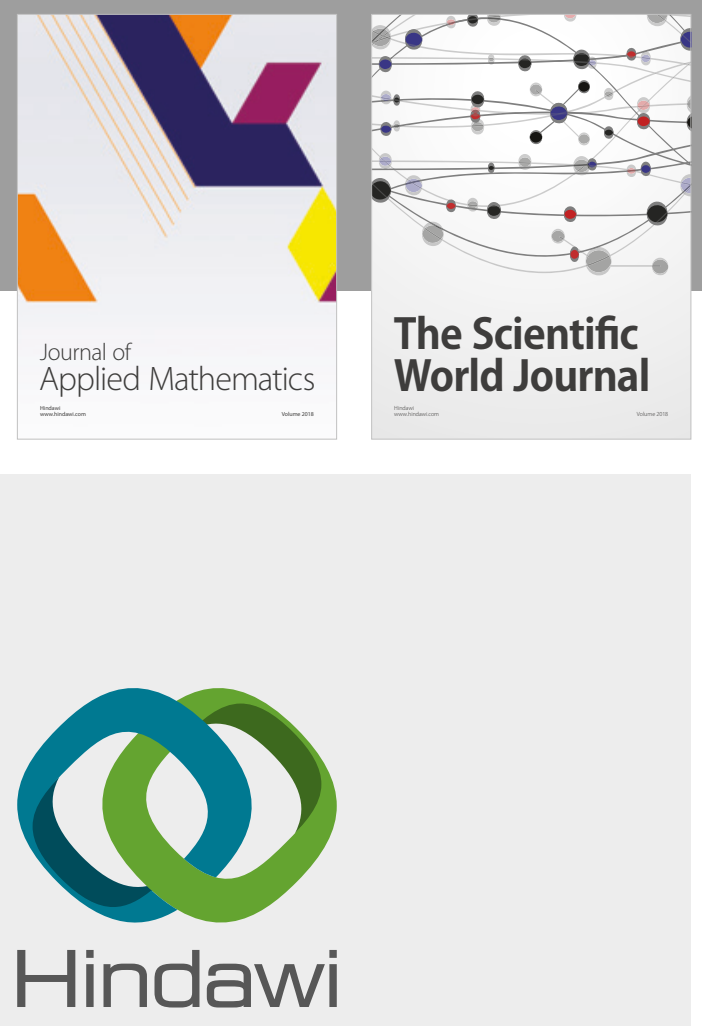

Submit your manuscripts at

www.hindawi.com

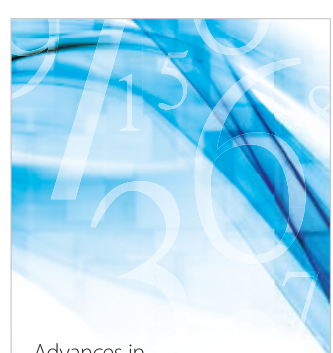

Advances in
Numerical Analysis
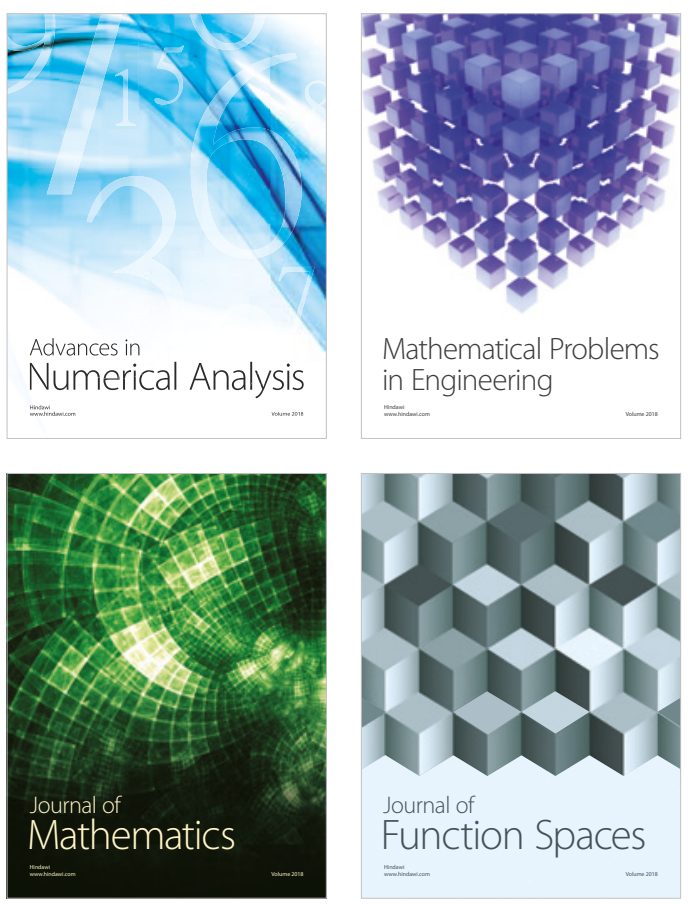

Mathematical Problems in Engineering

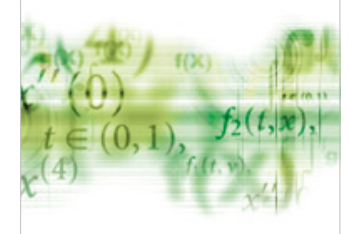

International Journal of

Differential Equations

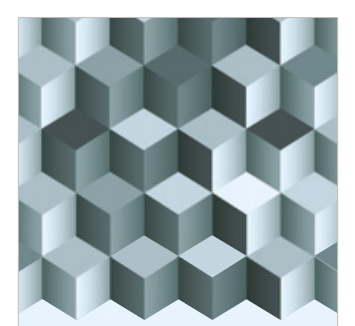

Journal of

Function Spaces
The Scientific

World Journal

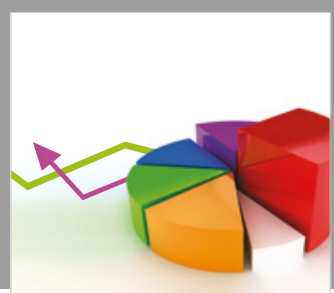

Journal of

Probability and Statistics
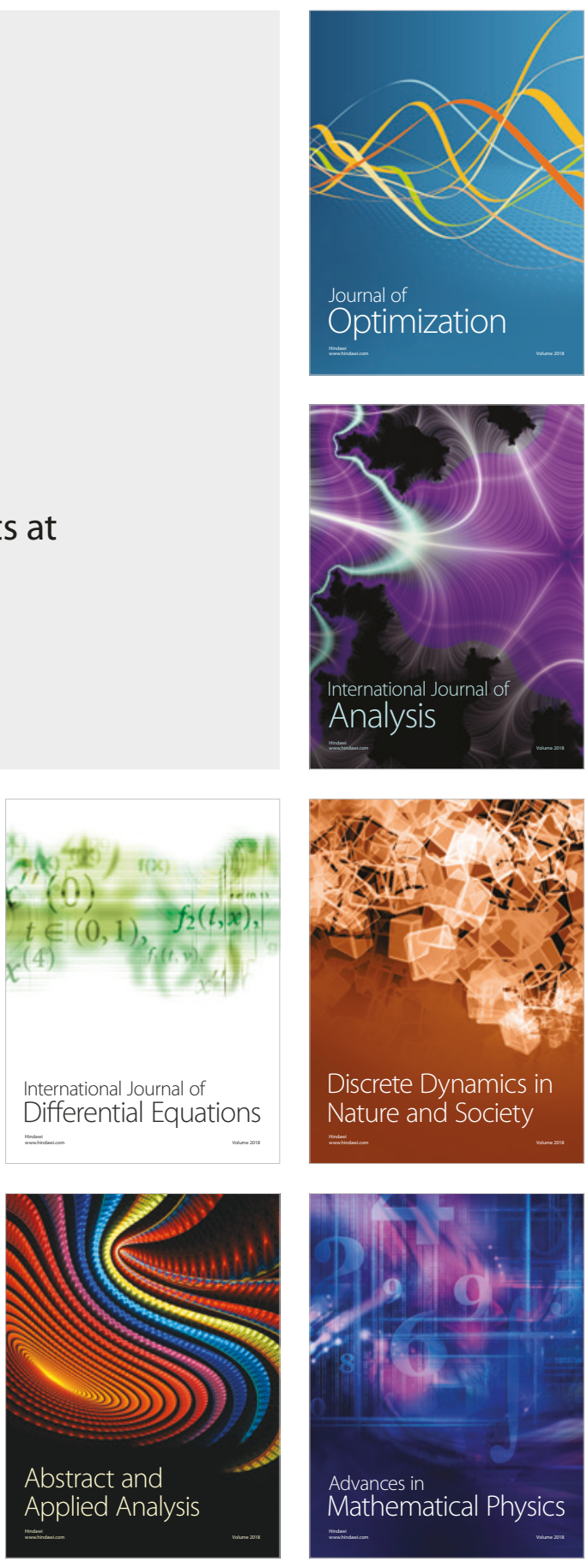